\title{
Trends in Clinical Information Systems
} Research in 2019

\section{An Overview of the Clinical Information Systems Section of the International Medical Informatics Association Yearbook}

\author{
W.O. Hackl1*, A. Hoerbst ${ }^{2 *}$, Section Editors for the IMIA Yearbook Section on Clinical \\ Information Systems \\ I Institute of Medical Informatics, UMIT - Private University of Health Sciences, Medical Informatics \\ and Technology, Hall in Tirol, Austria \\ 2 Medical Technologies Department, MCl - The Entrepreneurial School, Innsbruck, Austria
}

* Equal Contribution

\section{Summary}

Objective: To give an overview of recent research and to propose a selection of best papers published in 2019 in the field of Clinical Information Systems (CIS).

Method: Each year, we apply a systematic process to retrieve articles for the CIS section of the IMIA Yearbook of Medical Informatics. For six years now, we use the same query to find relevant publications in the CIS field. Each year we retrieve more than 2,000 papers. As CIS section editors, we categorize the retrieved articles in a multi-pass review to distill a pre-selection of 15 candidate best papers. Then, Yearbook editors and external reviewers assess the selected candidate best papers. Based on the review results, the IMIA Yearbook Editorial Committee chooses the best papers during the selection meeting. We used text mining, and term co-occurrence mapping techniques to get an overview of the content of the retrieved articles.

Results: We carried out the query in mid-January 2020 and retrieved a de-duplicated result set of 2,407 articles from 1,023 different journals. This year, we nominated 14 papers as candidate best papers, and three of them were finally selected as best papers in the CIS section. As in previous years, the content analysis of the articles revealed the broad spectrum of topics covered by CIS research.

Conclusions: We could observe ongoing trends, as seen in the last years. Patient benefit research is in the focus of many research activities, and trans-institutional aggregation of data remains a relevant field of work. Powerful machine-learningbased approaches, that use readily available data now often outperform human-based procedures. However, the ethical perspective of this development often comes too short in the considerations. We thus assume that ethical aspects will and should deliver much food for thought for future CIS research.

\section{Keywords}

Medical informatics; International Medical Informatics

Association; Yearbook; Clinical Information Systems

Yearb Med Inform 2020:121-8

http://dx.doi.org/10.1055/s-0040-1702018 issue of the IMIA Yearbook of Medical Informatics, we concluded that this trend was gaining momentum by the application of new or already known but, due to technological advances, now applicable methodological approaches. We had also found inspiring work that dealt with data-driven management of processes and the use of blockchain technology to support data aggregation beyond institutional boundaries [4].

These trends are ongoing in our recent analysis. We found a lot of high-quality contributions, but, on the other hand, we did not see outstanding innovations in the CIS field. As "Ethics in Health Informatics" is the special topic for the 2020 issue of the IMIA Yearbook of Medical Informatics, we intensified our focus on this topic when screening the CIS publications. But we had to realize that ethical aspects seem to be only a side issue as a research topic in the CIS domain.

\section{About the Paper Selection}

not just tools for health professionals. The patient increasingly moved in the center of research activities during the last years, and it has often been shown that CIS can create significant benefits for patients.

So, during the last years, we identified the trend of moving away from clinical documentation to patient-focused knowledge generation and support of informed decision. In the analysis performed in the previous
The selection process used in the CIS section is stable now for six years. We described it in detail in [3], and the full queries are available upon request.

We carried out the queries in mid-January 2020. This year, the CIS search result set comprised 2,407 unique papers. From these papers, we retrieved 2,143 from PubMed
Trans-institutional information exchange and data aggregation are vital research 
and found 264 additional publications (de-duplicated) in Web of Science ${ }^{\mathrm{TM}}$. The resulting articles have been published in 1,023 different journals. Table 1 depicts the Top-15 journals with the highest numbers of resulting articles.

Again, we used RAYYAN, an online systematic review tool [5], to carry out the multi-pass review. As section editors, we both (WOH, $\mathrm{AH}$ ) independently reviewed all 2,407 publications. Ineligible articles were excluded based on their titles and/or abstracts (WOH: $\mathrm{n}=2,323 ; \mathrm{AH}: \mathrm{n}=2,376$ ). The agreement between the two editors was $\mathrm{n}=2,308$ for "exclude", and $\mathrm{n}=16$ for "not exclude" (i.e., include). We calculated an agreement rate of $96.6 \%$ (Cohen's kappa 0.26 ) for this assessment. We solved the remaining 83 conflicts on mutual consent, which resulted in nine additional inclusions.

The final candidate best papers selection from the remaining 25 publications was done based on full-text review and yielded 15 candidate best papers published in 2019 . We then had to remove one paper as it also had been selected as a candidate best paper for the Decision Support Systems section of the IMIA Yearbook. For each of the remaining candidate best papers, at least five independent reviews were collected. Due to COVID-19 restrictions, the selection meeting of the IMIA Yearbook Editorial Committee was held as a videoconference on Apr 17, 2020. In this meeting, three papers [6-8] were finally selected as best papers for the CIS section (Table 2). A content summary of these three CIS best papers can be found in the appendix of this synopsis.

\section{Findings and Trends in 2019}

As section editors, we get a broad overview of the research field of the CIS section during the selection of the best papers. As this overview may be biased and to avoid selective perception, as in the previous years [1-4], we additionally apply a more formal text mining and bibliometric network visualizing approach [9] to summarize the content of titles and abstracts of the articles in the CIS result set.

Table 1 Number of retrieved articles for Top-15 journals.

Journal (Total Number of Journals $=957$ ) Number of papers

\begin{tabular}{|c|c|}
\hline PLOS ONE & 51 \\
\hline JOURNAL OF MEDICAL INTERNET RESEARCH & 47 \\
\hline INTERNATIONAL JOURNAL OF MEDICAL INFORMATICS & 43 \\
\hline HEALTH COMMUNICATION & 41 \\
\hline BMJ OPEN & 34 \\
\hline BMC MEDICAL INFORMATICS AND DECISION MAKING & 33 \\
\hline COMPUTERS, INFORMATICS, NURSING: CIN & 33 \\
\hline INTERNATIONAL JOURNAL OF ENVIRONMENTAL RESEARCH AND PUBLIC HEALTH & 30 \\
\hline JOURNAL OF MEDICAL SYSTEMS & 26 \\
\hline HEALTH INFORMATICS JOURNAL & 26 \\
\hline JOURNAL OF THE AMERICAN MEDICAL INFORMATICS ASSOCIATION: JAMIA & 25 \\
\hline BMC HEALTH SERVICES RESEARCH & 20 \\
\hline APPLIED CLINICAL INFORMATICS & 18 \\
\hline JMIR MHEALTH AND UHEALTH & 18 \\
\hline VACCINE & 17 \\
\hline PATIENT EDUCATION AND COUNSELING & 16 \\
\hline DRUG SAFETY & 14 \\
\hline ENVIRONMENTAL MONITORING AND ASSESSMENT & 14 \\
\hline JMIR MEDICAL INFORMATICS & 14 \\
\hline RESEARCH IN SOCIAL \& ADMINISTRATIVE PHARMACY: RSAP & 13 \\
\hline BMJ HEALTH \& CARE INFORMATICS & 12 \\
\hline ENVIRONMENTAL SCIENCE AND POLLUTION RESEARCH INTERNATIONAL & 12 \\
\hline EXPERT OPINION ON DRUG SAFETY & 12 \\
\hline INFORMATICS FOR HEALTH \& SOCIAL CARE & 12 \\
\hline JOURNAL OF BIOMEDICAL INFORMATICS & 12 \\
\hline
\end{tabular}

Table 2 Best paper selection of articles for the IMIA Yearbook of Medical Informatics 2020 in the section 'Clinical Information Systems'. The articles are listed in alphabetical order of the first author's surname.

Section

Clinical Information Systems

- Gordon WJ, Wright A, Aiyagari R, Corbo L, Glynn RJ, Kadakia J, Kufahl J, Mazzone C, Noga J, Parkulo M, Sanford B, Scheib $P$, Landman AB. Assessment of employee susceptibility to phishing attacks at US health care institutions. JAMA Netw open 2019;2(3):e190393.

- Hill BL, Brown R, Gabel E, Rakocz N, Lee C, Cannesson M, Baldi P, Loohuis LO, Johnson R, Jew B, Maoz U, Mahajan A, Sankararaman S, Hofer I, Halperin E. An automated machine learning-based model predicts postoperative mortality using readily-extractable preoperative electronic health record data. Br J Anaesth 2019;123(6):877-86.

- Shen N, Bernier T, Sequeira L, Strauss J, Silver MP, Carter-Langford A, Wiljer, D. Understanding the patient privacy perspective on health information exchange: A systematic review. Int J Med Inform 2019;125:1-12.

As in the past year, we extracted the authors' keywords ( $\mathrm{n}=18,962)$ from all articles and present their frequency in a tag cloud (Figure 1). We found 6,796 different keywords, of which 4,956 were only used once. As in the previous year, most frequent keywords were "human" ( $n=733)$, followed by "female" $(\mathrm{n}=357)$, "electronic health record(s)" (n=344), "male" (n=334), "adult" $(\mathrm{n}=226)$, "middle aged" $(\mathrm{n}=218)$, and "health communication" ( $\mathrm{n}=188)$.

The bibliometric network reveals more details on the content of the CIS publications. Figure 2 depicts the resulting co-occurrence 


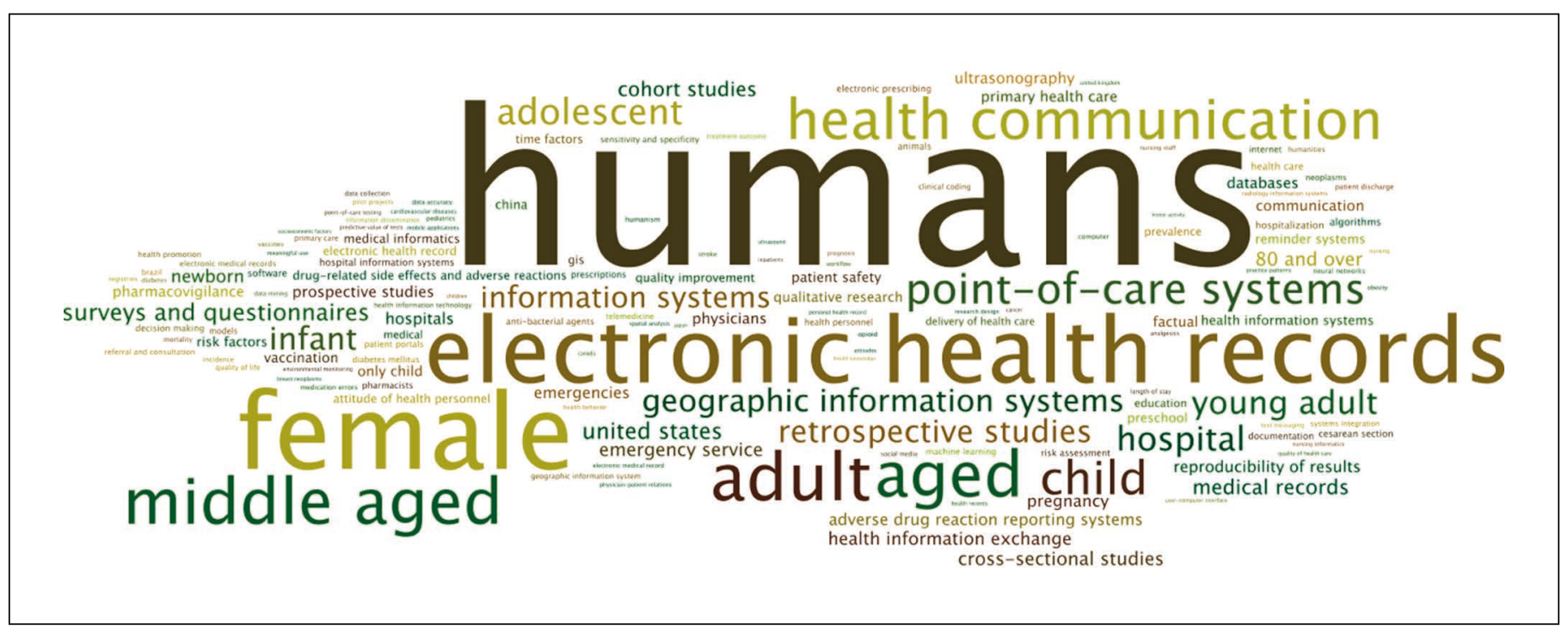

Fig. 1 Tag cloud illustrating the frequency of authors' keywords (only top keywords out of $n=6,796$ are shown) within the 2,407 papers from the CIS query result set. Font size corresponds to frequency (most frequent keyword was "humans" $n=733$ ).

map of the top-1000 terms $(\mathrm{n}=1,087$, most relevant $60 \%$ of the terms) from the titles and abstracts of the 2,407 papers of the CIS result set. The cluster analysis of titles and abstracts yielded five clusters. The two most massive clusters, the yellow one on the right side with 337 items and the green one on the left with 334 items, describe some context factors from the studies. Whereas the yellow cluster seems to represent an intramural view with "hospital record" as a prominent item, the green cluster represents the trans-institutional perspective of CIS with "health record" as a prominent item.

All three of the best papers in the CIS section can be assigned to these two clusters. The contribution by Brian L. Hill and colleagues [8], who successfully created a fully automated machine-learning-based model for postoperative mortality prediction is in the yellow cluster. We selected this paper from the British Journal of Anaesthesia as the approach is innovative, uses only preoperative available medical record data, and can better predict in-hospital mortality than other state-of-the-art methods. We also selected it because, on the other hand, we believe that there is a need for discussion from an ethical point of view when an automated approach "decides" on the "eligibility" of patients for specific treatments. So, it perfectly fits in the special topic "Ethics in Health Informatics" of the IMIA Yearbook 2020.

The next of the best papers can be assigned to the green cluster. Nelson Shen and colleagues conducted a systematic review that helps to better understand an essential aspect of health information exchange: the patient privacy perspective [6]. This contribution is also interesting given this IMIA Yearbook edition's special topic.

The last of the best papers comes from William J. Gordon and colleagues [7], who investigated health care employees' susceptibility to phishing attacks. This study can be assigned to both clusters, and should all of us remember that cybersecurity is increasingly critical and should be tackled accordingly.

From the remaining candidate best papers, we can assign a reasonable proportion to the two main clusters. To the green cluster, we can assign four candidate best papers. Esmaeilzadeh and colleagues investigated the effects of data entry structure on patients' perceptions of information quality in health information exchange [11]. The proportion of "blockchain papers" is slightly growing. We thus again selected one systematic review, a paper by Vazirani and colleagues [12], as an excellent read to dive into this emerging field and to learn about the applicability of this technology in healthcare, health record management, and health information exchange. Trust is also an essential aspect of health information exchange. Therefore, we selected a paper of Um and colleagues who designed a trust information management framework for Social Internet of Things Environments [13]. Although this paper has no obvious and direct connection to the health care system at first glance, we find it describes an interesting approach that can advance the realization of trustful health services, which always have to exchange data to some extent. The next paper in this cluster comes from Kim and colleagues who propose an ontology and a simple classification scheme for clinical data elements based on semantics [14].

Three candidate best papers represent the intramural perspective in the yellow cluster. This perspective often also includes a patient safety aspect. Thomas and colleagues report on the use of digital facial images in a children's hospital to confirm patient identity before anesthesia to increase patient safety [15]. The next candidate paper in this cluster comes from 


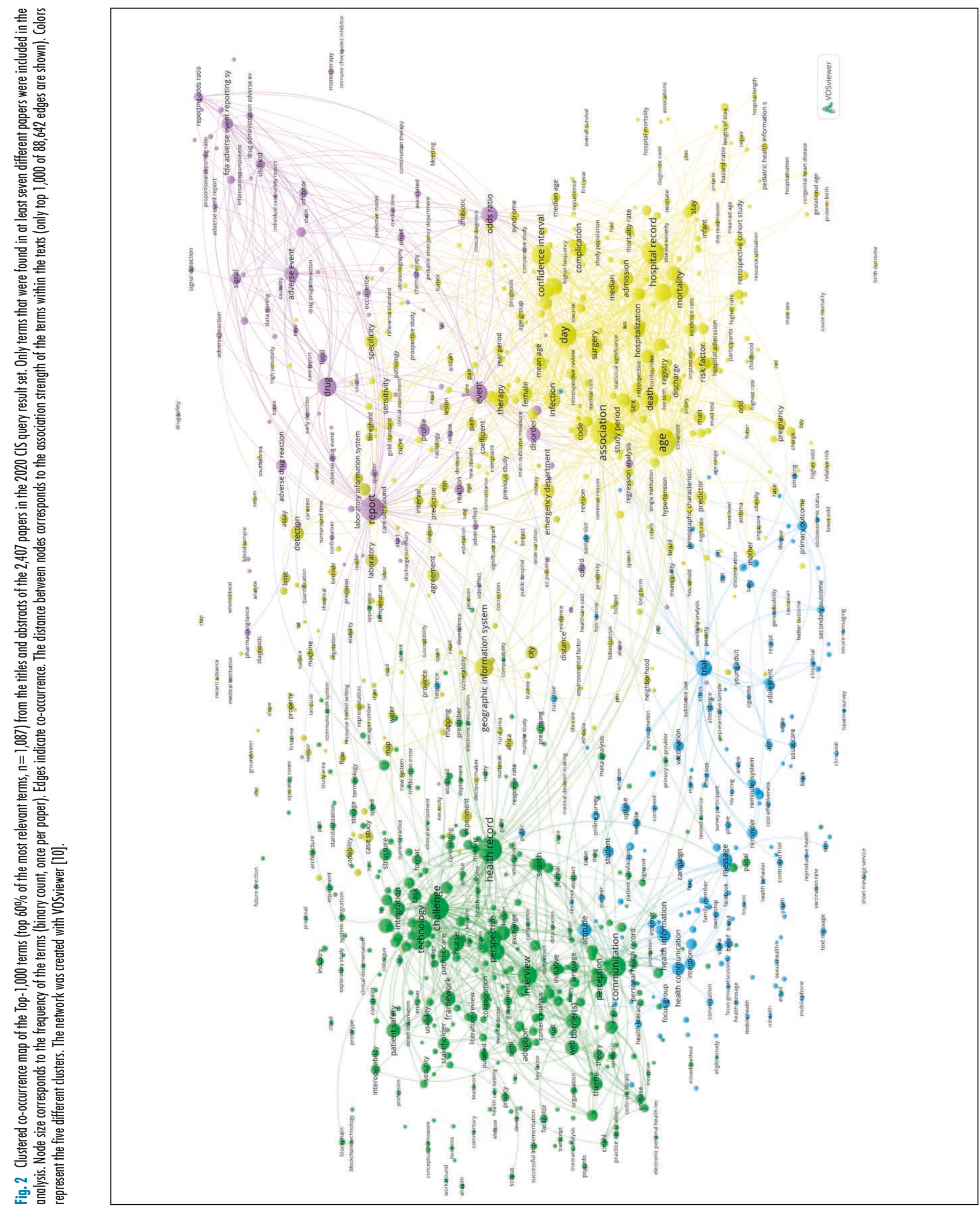


Signaevsky and colleagues who show how a deep-learning-based approach can help to improve diagnostic assessments in neuropathology [16]. The third "yellow" candidate paper comes from Bernard and colleagues [17]. They present a very inspiring visualization technique for representing multiple patient histories and their course over time in graphical dashboard networks. The development process of these dashboards is well described and can give valuable hints to all who are interested in dashboard design.

The golden cluster in the middle (143 items) with the term "geographic information system" as central hub divides the two main clusters. The blue cluster (bottom left, 162 items) mainly holds items from the studies' objectives, target measures, and methods sections. The purple cluster on the top right (111 items) is continuously present over the years. It contains items that are associated with adverse events and patient safety research.

An assignment to one of these clusters is difficult for the rest of the candidate papers. However, they have one aspect in common. They address various ethical aspects that are relevant in the CIS field. Sure, for the most part, these aspects are not explicitly mentioned in the papers. Nevertheless, we want to present them and put it in the hands of the reader to think about.

The first of the papers in this group comes from Blijleven and colleagues who developed a framework for the sociotechnical analysis of electronic health system workarounds [18]. Very inspiring. The next one, a paper on ethical and regulatory considerations for using social media platforms to locate and track research participants by Bhatia-Lin and colleagues in the American Journal of Bioethics [19], also made us think a lot. The next one, a position paper from Steil and colleagues in Methods of Information in Medicine [20], brought our thoughts in a completely different direction. Every reader who has wondered how the use of robotic systems in the operating room can or will bring new forms of team-machine interaction should put this paper to their reading list. To complete our selection, we want to direct the light to the dark side of CIS and health information technology (HIT), which also exists, no question. Gardner and colleagues surveyed physicians on their HIT use. More than a quarter of the $>1,700$ respondents reported burnout and 70\% reported HIT-related stress [21]. We think this is also an ethical CIS aspect worth considering.

As every year, at the very end of our review of findings and trends for the clinical information systems section, we want to recommend a reading of this year's survey article in the CIS section by Ursula Hübner, Nicole Egbert and Georg Schulte. They investigated ethical aspects in recent CIS research in more detail [22].

\section{Conclusions and Outlook}

As in the previous years, we could observe major trends being further continued. These trends include research about the actual benefits of patients with regard to health information exchange and their active participation in healthcare. Another trend that now remained valid for several years is the trans-institutional aggregation of data. It seems that the challenges around this topic are still not sufficiently solved. However, we could observe an ongoing shift away from fundamental technical problems to more content/context-related questions of data aggregation. The observed popularity of machine-learning approaches on readily available clinical data sets such as Electronic Health Record data in our 2019 analysis seems to increase, especially, their application in supporting clinical processes such as risk assessment or the proactive implementation of interventions. However, ethical aspects are, in many cases, not considered at all or are only regarded as a peripheral topic. These aspects leave a broad gap for further investigations.

\section{Acknowledgments}

We would like to acknowledge the support of Lina Soualmia, Adrien Ugon, Brigitte Séroussi, Martina Hutter, and the whole Yearbook Editorial Committee as well as the numerous reviewers in the selection process of the CIS best papers.

\section{References}

1. Hackl WO, Ganslandt T. New Problems - New Solutions: A Never Ending Story. Findings from the Clinical Information Systems Perspective for 2015. Yearb Med Inform 2016;(1):146-51.

2. Hackl WO, Ganslandt T. Clinical Information Systems as the Backbone of a Complex Information Logistics Process: Findings from the Clinical Information Systems Perspective for 2016. Yearb Med Inform 2017;26(1):103-9.

3. Hackl W, Hoerbst A. On the Way to Close the Loop in Information Logistics: Data from the Patient Value for the Patient. Yearb Med Inform 2018 Aug 29;27(01):91-7.

4. Hackl, WO, Hoerbst A. Managing Complexity. From Documentation to Knowledge Integration and Informed Decision Findings from the Clinical Information Systems Perspective for 2018. Yearb Med Inform 2019 Aug 16;28(01):95-100.

5. Ouzzani M, Hammady H, Fedorowicz Z, Elmagarmid A. Rayyan-a web and mobile app for systematic reviews. Syst Rev 2016;5(1):210.

6. Shen N, Bernier T, Sequeira L, Strauss J, Silver MP, Carter-Langford A, et al. Understanding the patient privacy perspective on health information exchange: A systematic review. Int J Med Inform 2019;125:1-12.

7. Gordon WJ, Wright A, Aiyagari R, Corbo L, Glynn RJ, Kadakia J, et al. Assessment of Employee Susceptibility to Phishing Attacks at US Health Care Institutions. JAMA Netw open 2019;2(3):e190393.

8. Hill BL, Brown R, Gabel E, Rakocz N, Lee $\mathrm{C}$, Cannesson $\mathrm{M}$, et al. An automated machine learning-based model predicts postoperative mortality using readily-extractable preoperative electronic health record data. Br J Anaesth 2019;123(6):877-86.

9. Waltman L, van Eck NJ, Noyons ECM. A unified approach to mapping and clustering of bibliometric networks. J Informetr 2010;4(4):629-35.

10. van Eck NJ, Waltman L. Software survey: VOSviewer, a computer program for bibliometric mapping. Scientometrics 2010;84(2):523-38.

11. Esmaeilzadeh P, Mirzaei T, Maddah M. The effects of data entry structure on patients' perceptions of information quality in Health Information Exchange (HIE). Int J Med Inform 2019;135:104058.

12. Vazirani AA, O'Donoghue O, Brindley D, Meinert E. Implementing Blockchains for Efficient Health Care: Systematic Review. J Med Internet Res 2019;21(2):e12439.

13. Um T-W, Lee E, Lee GM, Yoon Y. Design and Implementation of a Trust Information Management Platform for Social Internet of Things Environments. Sensors (Basel) 2019;19(21).

14. Kim HH, Park YR, Lee KH, Song YS, Kim JH. Clinical MetaData ontology: a simple classification scheme for data elements of clinical data based on semantics. BMC Med Inform Decis Mak 2019;19(1):166.

15. Thomas JJ, Yaster M, Guffey P. The Use of Patient Digital Facial Images to Confirm Patient Identity in a Children's Hospital's Anesthesia Information Management System. Jt Comm J Qual patient Saf 
Hackl et al.

2020;46(2):118-21.

16. Signaevsky M, Prastawa M, Farrell K, Tabish N, Baldwin E, Han N, et al. Artificial intelligence in neuropathology: deep learning-based assessment of tauopathy. Lab Invest 2019 Jul;99(7):1019-29.

17. Bernard J, Sessler D, Kohlhammer J, Ruddle RA. Using Dashboard Networks to Visualize Multiple Patient Histories: A Design Study on Postoperative Prostate Cancer. IEEE Trans Vis Comput Graph 2019;25(3):1615-28.

18. Blijleven V, Koelemeijer K, Jaspers M. SEWA: A framework for sociotechnical analysis of electronic health record system workarounds. Int J Med Inform 2019;125:71-8.

19. Bhatia-Lin A, Boon-Dooley A, Roberts MK,
Pronai C, Fisher D, Parker L, et al. Ethical and Regulatory Considerations for Using Social Media Platforms to Locate and Track Research Participants. Am J Bioeth 2019 Jun;19(6):47-61.

20. Steil J, Finas D, Beck S, Manzeschke A, Haux R. Robotic Systems in Operating Theaters: New Forms of Team-Machine Interaction in Health Care. Methods Inf Med 2019 Jun;58:e14-25.

21. Gardner RL, Cooper E, Haskell J, Harris DA, Poplau S, Kroth PJ, et al. Physician stress and burnout: the impact of health information technology. J Am Med Inform Assoc 2019;26(2):106-14.

22. Huebner UH, Egbert N, Schulte G. Clinical Information Systems - Seen through the Ethics Lens. Yearb Med Inform 2020:104-14.

\section{Correspondence to}

Dr. Werner 0 Hackl Institute of Medical Informatics

UMIT - Private University for Health Sciences, Medical Informatics and Technology

Eduard-Wallnoefer-Zentrum 1

6060 Hall in Tirol, Austria

Tel: + 435086483806

E-mail: werner.hackl@umit.at

Dr. Alexander Hörbst

Medical Technologies Department

MCI - THE ENTREPRENEURIAL SCHOOL

Universitätsstrasse 15

6020 Innsbruck, Austria

Tel: +43512 20704410

E-mail: alexander.hoerbst@mci.edu 
Appendix: Content Summaries of Selected Best Papers for the IMIA Yearbook 2020 Section "Clinical Information Systems"

\section{Gordon WJ, Wright A, Aiyagari R, Corbo L, Glynn RJ, Kadakia J, Kufahl J, Mazzone C, Noga J, Parkulo M, Sanford B, Scheib P, Landman $A B$}

\section{Assessment of employee susceptibility to phishing attacks at US health care institutions}

\section{JAMA Netw open 2019;2(3):e190393}

The current paper from Gordon et al., picks up on an important topic from the field of data security and investigates the susceptibility of healthcare employees to phishing attacks in the US. The recent past has shown that attackers have increasingly targeted healthcare organizations, not only with substantial economic impact but also with a strong influence on patient treatment. The authors illustrate multiple examples ranging from partial unavailability of systems up to a two-week complete shutdown of systems. The authors have carried out an investigation to get an insight into the reasons why employees of healthcare organizations fall victims of phishing campaigns. The investigation is based on a retrospective, multicenter quality improvement study that included six US healthcare organizations that represent the entire spectrum of care and a range of US geographies. All organizations have an information security program in place. The respective organizations have carried out phishing simulations in their facilities in the past, based on vendor- or custom-developed software tools.

Data about the phishing attacks were collected from the different institutions, and emails were classified according to their content in three categories: office-related, personal, or information technology-related. Several statistical values were calculated, such as the click rates, median click rates, and odds ratios (with 95\% CI). Correlation was, amongst others, computed for the year, number of campaigns, email category, and season. In total, the data set included $95 \mathrm{cam}$ paigns with emails sent from 2011 to 2018. The overall click rate across all institutions and campaigns was $14.2 \%$, although the authors observed considerable differences in the click rate of institutions ranging from $7.4 \%$ to $30.7 \%$. The authors found out that repeated phishing campaigns were associated with decreased odds of clicking on a subsequent phishing email.

Interestingly, the year is not significantly associated with the click rate. Further, emails that were related to the personal email category had a significantly higher probability of being clicked. The same is true for seasons, both the spring and summer seasons were associated with higher click rates. In models adjusted for several potential confounders, including year, the institutional campaign number, institution, and email category, the odds of clicking on a phishing email were 0.511 lower for six to ten campaigns at an institution and 0.335 lower for more than ten campaigns at an institution. The study could well demonstrate that the healthcare domain compares well to other industries and that employees benefit from education, training, and that experiences made from other simulated phishing campaigns can help employees to stay aware. In addition, the healthcare domain has some particularities that make it especially vulnerable to attacks such as turnover of employees, endpoint complexity, or information system interdependence. It is therefore inevitable that all participants in the healthcare domain understand these security risks, particularly as safe and effective health care delivery involves more and more information technology.

\section{Hill BL, Brown R, Gabel E, Rakocz N, Lee C, Cannesson M, Baldi P, Loohuis LO, Johnson R, Jew B, Maoz U, Mahajan A, Sankararaman S, Hofer I, Halperin E}

An automated machine learning-based model predicts postoperative mortality using readily-extractable preoperative electronic health record data

\section{Br J Anaesth 2019;123(6):877-86}

The majority of surgical complications is associated with a small group of high-risk patients. Often these patients would substan- tially benefit from early identification of their high-risk of potential complications, as proactive, early interventions can help reduce or even avoid perioperative complications. Existing approaches to this problem either require a clinician to review a patient's chart such as the American Society of Anesthesiologists (ASA) physical status classification or lack specificity. The work of Hill et al., is dedicated to the investigation of a machine learning approach that uses readily available patient data for the prediction of certain risks and takes changing patient conditions into account. Data from 53,097 surgical patients (2.01\% mortality rate) who underwent general anesthesia between 2013 and 2018 were collected from the perioperative data warehouse at UCLA Health to populate a series of 4,000 distinct measures and metrics. In the next step, classification models to predict in-hospital mortality as a binary outcome were trained (model endpoint) and the outcome for a subset of patients was checked with trained clinicians. For the actual creation and training of models, four different classification models, logistic regression, ElasticNet, random forests, and gradient boosted trees were evaluated. The performance of the created models was then compared with existing clinical risk scores such as the ASA score, POSPOM score, and Charlson comorbidity score. The mean value of the area under the receiver operating characteristic (AUROC) curve $(95 \%$ CI, 1,000 predictions) was used to compute the performance. When using the ASA status or the Charlson comorbidity score as the only input features, the linear models (logistic regression, ElasticNet) outperform the non-linear models (random forest, XGBoost). However, for the other feature sets, the non-linear models outperform the linear models. In particular, the random forest has the highest AUROC compared with the other models. The authors were able to show that a fully automated preoperative risk prediction score can better predict in-hospital mortality than the ASA score, the POSPOM score, and the Charlson comorbidity score. Unlike previously developed models, the results also indicate that the inclusion of the ASA score in the model did not improve the predictive ability. Another advantage of such an automated model is that it allows for the 
continuous recalculation of risk longitudinally over time. However, the authors also state several limitations of the study such that the incidence of mortality in the testing set was less than $2 \%$, implying that a model that blindly reports 'survives' every time will have an accuracy greater than 98\%. Nonetheless, the model outperforms current major models in use.

\section{Shen N, Bernier T, Sequeira L, Strauss J, Silver MP, Carter-Langford A, Wiljer, D}

\section{Understanding the patient privacy perspective on health information exchange: A systematic review}

\section{Int J Med Inform 2019;125:1-12}

The exchange of health information and the ability to share information regarding the patient and treatment has become an essential element in the effective and efficient provision of healthcare services. On the other side, these developments have also led to increasing concerns by patients not being able to properly control these information flows. Although privacy concerns are often quoted in publications regarding the exchange of health information, they are seldom investigated with regard to their influence on the patient-provider relationship in healthcare. The paper of Shen et al., focuses on an indepth exploration of the patient's perspective towards privacy in the context of health information exchange. For this reason, the authors have conducted a systematic review, which was based on PRISMA and aimed at providing a conceptual synthesis of the patient privacy perspective and its associated antecedents and outcomes; identify gaps in the APCO model (Antecedent, Privacy Concern, Outcomes macro-model) and describe the current state of privacy research. The APCO model was developed by the authors in advance to the present study. Major databases were queried for empirical studies focused on patient/public privacy perspectives in the context of HIT that were published between 2015 and 2017. Data was extracted based on the elements of the APCO model, and subsequently, new elements were added if outside the APCO model. The authors found 39 quantitative, 15 qualitative, and five mixed-methods studies that were relevant. The analysis of the antecedent factors with regard to their influence on patient privacy concerns showed a mixed picture, and an evident positive or negative influence was often not deducible, or the number of studies was low. The authors assumed income, political ideology, and quality of care as being agreed upon studies. The same is true for privacy concerns related to outcome factors, where the authors assume a willingness to share, protective behaviors, benefits, and risks, as agreed by the studies found. Summarizing, the patient privacy perspective seems to be of dynamic and nuanced meaning that is strongly dependent on its context. So, it is difficult to characterize the patient perspective, although privacy concerns, as such, are found in many studies and are expressed as a serious concern. This may also be because many studies have analyzed the concept of privacy only as a peripheral topic and have distilled privacy into a single question. The authors plead that future research needs to place greater emphasis on understanding how antecedent factors can alleviate privacy concerns, build trust, and empower patients. In addition, they claim that building a base of evidence on the actual effects of privacy concerns will help to reduce value-laden discussions and normative assumptions. 